\title{
An Sithean Ruadh
}

\section{Author(s): Allan MacDonald}

Source: The Celtic Review, Vol. 3, No. 9 (Jul., 1906), pp. 77-83

Stable URL: http://www.jstor.org/stable/30069899

Accessed: 09-06-2016 05:30 UTC

Your use of the JSTOR archive indicates your acceptance of the Terms \& Conditions of Use, available at

http://about.jstor.org/terms

JSTOR is a not-for-profit service that helps scholars, researchers, and students discover, use, and build upon a wide range of content in a trusted digital archive. We use information technology and tools to increase productivity and facilitate new forms of scholarship. For more information about JSTOR, please contact support@jstor.org. 


\section{AN SITHEAN RUADH}

\section{By the late Fr. Allan MacDonald of Eriskay}

I

Beadagan I. 'Bodach na croite! Bodach na croite!' (a' teicheadh).

Domhnull Crotach. 'Bodach na croite! Bodach na croite!' Port nam beadagan; port nam beadagan. Nach gòrach mi toirt feairt orra?

Beadagan II. 'Bodach na croite! Stob anns a' phoit i!' (a' teicheadh).

Domhnull. Ni thu rud math fhathasd, a laochain, ma leigear leat. Mac $t$ ' athar 's mac do mhathar gu dearbh! Cha 'n ionnsuichear modh ann an nead feannaige!

Thug e chasan leis, 's tha chead aige. Bho mhoch gu dubh 'gam bhodhradh leis an aon duan ud, cha b'ioghnadh ged a dh' fhdsainn searbh dhith.

Och! och! Cha robh mi riamh cho anmoch a' dol dhachaidh, 's gu dearbh fhein bu cheart cho math leam an Sithean Ruadh a bhi as mo dheaghaidh 's a bhi rombam. Cha 'n e gu 'm faca mise dad riamh timchioll air, ach mur a faca chunnaic daoine eile agus chuala.

$\mathrm{O}$ m' fheudail mhor! Nach ann dhomh a dh' éirich! Nach seall sibhse an $t$-suil bheag soluis anns a' chnoc! Carson a thug mi iomradh riamh air? Ga bri (cia air bith) mar dh' eireas dhomhsa cha 'n 'eil saod agam air faighinn seachad gun a dhol a stigh. ' $\mathrm{S}$ buidhe dhomh codhiù a' chorrag bheag chruadhach a bhi air mo ghiùlan. Cha 'n fhaigh iad a' chomhla dhruideadh orm ma 's urrainn domhsa.

II

Domhnull (a' gabhail a stigh). Gu 'm bu slàn do 'n tigh 's do 'n chuideachd.

Bean-Shith. Gu 'm bu slàn dhuibhse, a dhuine chòir, agus làn di-ur-beatha do 'n t-Sithean Ruadh. Teannaibh a nuas, is dianaibh suidhe a bhos goirid dhomb fhein.

Domhnull. Tapadh leibhse, a bhean-uasal, ach tha mi cho math 's a dh' iarruinn far a bheil mi. 
$B$. S. Cha b' e cùl na combladh aite aon duine a thigeadh a chur seachad greis de 'n oidhche combla ruinn. Teannaibh a nuas; teannaibh a nuas.

Domhnull. B' olc a fhreagradh e dhomhsa ar diultadh, a bhean uasal; agus is mise ni sin suidhe ri ' $r$ taobh, 's a chl mi fhein sona dheth an cead fhaighinn.

$B$. S. Tha cathair mhor shocrach an so.

Domhnull. Nach i tha math?

$B$. $S$. Co dhiubh b' fhearr leibh tarruinn a thoirt air biadh, no air ceol is aighear an toiseach.

Domhnull. Bidh roghainn a' Ghàidheil agamsa; bhithinn $a^{\prime}$ cluinntinn thall nach robh na b' fhearr gu cedl is aighear is dannsa na sibhse, agus b' fhior-mhiannach leam, a' chuideachda bhoidheach a tha timchioll fhaicinn air an ùrlar, na 'm b' $e$ an toil e.

B. $S$. Cha do dh' iarr iad riamh ciuird a roghainn, agus b' olc a thigeadh e dhuinn mur dianamaid uiread sin, gu beagan toileachaidh a thoirt dhuibh.

Domhnull. Tapadh leibhse. 'S mi tha 'n ar comain.

B. S. (Smeid i air na sithichean agus leam iad thun an ùrlair.) C'àite bheil thu, a Mhurchaidh? Co sheinneas an cedl ma tha thusa dol a dhannsa?

Murchadh. Bha dùil leam gu'n robh Fionnladh aig an tigh. 'S e thug orm éirigh. Ach gabhaidh mise port a chumas air an ùrlar gu maduinn iad.

B. S. Gabhaidh tu'm port mar as fhearr a theid agad air, $m u$ 'm bi 'n duine coir a thainig dhachaidh a' fanaid ort.

Murch. 'S mise ni sin, ach feumaidh a h-uile h-anam a th' air an ùrlar togail fo 'n fhonn.

B. S. 'Mar sin fhein e,' mar a bha Ciogain an t-saoghail ud eile 'g radh. Siuthadaibh ma ta. (Tha iadsan a' leum thun an ùrlair.) A Mhurchaidh, suas am port!

Murch.

Di-luain Di-mairt.

Di-luain Di-mairt.

$A^{\prime}$ Chuideachd. Di-luain Di-mairt.

Di-luain Di-mairt.

(Na sithichean a' dannsa fad an t-siubhail.)

$B$. S. Ciamar a tha chùis a' cordadh ribh ? 
Domhnull. Dannsa na bu chridheala na sin cha 'n fhaca mi bho rugadh mi. Ach, an cead duibhse, tha mi 'm beachd gu 'm biodh an danns' fhein a dhà fheobhas na 'm biodh am port air a leasachadh.

B. S. Am portair a leasachadh ! Co chuala riamh iomradh air a leithid? Na chunnaic sinne riamh a' toirt ionnsuidh air a leithid 's ann a bha iad a' fagail na cùise a dha mhiosad 's a fhuaradh.

Domhnull. Chunnaic mise a' chaochladh sin corra uair, agus 's e sin a tha toirt misneach dhomh tarruinn a thoirt air.

$B$. S. An teid agaibh fhein, an saoil sibh, air a' ghnothach a chur air dòigh?

Domhnull. Cha mhath leam a ràdh gu 'n teid, 's cha mhath leam a ràdh nach teid. Ach ma's e ur toil fhein e, bheir mi ionnsaidh air; is gabhaidh sibh mo leisgeul mur teid an gnothach leam, 's nach robh a' bhàrdachd a' ruith anns na daoine bho 'n tainig mi.

Tromhad a nall, a Mhurchaidh, is fiach ciamar a fhreagras so :-

Di-luain Di-mairt.

Di-luain Di-mairt.

Di-luain Di-mairt Di-ciadaoin.

Murchadh. Di luain Di-mairt.

Di-luain Di-mairt.

Di luain Di-mairt Di-ciadaoin.

A' Chuideachd. Di luain Di-mairt.

Di-luain Di-mairt.

Di luain Di-mairt Di-ciadaoin.

(Air am bonnan bha iad; 's cha robh dannsa na port aci riamh thun an nochd. Nuair a sguir iad chruinnich iad uile mu Dhomhnull.)

B. S. Port cho ruithteach 's cho siùbhlach sud, cha chualas riamh ann an Sithean mor a' Chrimleid fhein. Iarraibh ur roghainn's ur dà roghainn, a dhuine chòir, rath na ealain, no ealain is rath. Sibh fhein a b' fhiach sin.

Domhnull. Tha dh' ealain 's a rath agam cheana na dh' fhoghnas dhomh ri m' mhaireann; ach na 'm biodh saod 
agaibh air a dhianamh, 's nam faiceadh sibh fhein iomchaidh, bhithinn fada 'n ar comain na 'n togadh sibh an cnap so bharr mo dhroma. Bidh beadagain nam bailtean a' balachd orm an comhnaidh air a shàilleabh.

B. S. Suidheadh sibhse a bhos an so 'nam chathair fhein is gu 'm buail mi an slacan-sithe oirbh, 's bithidh sibh cho deas direach ri aois fichead bhiadhna.

(Thuit a' chroit dheth, is shadadh a nunn gu iomall an tSithein i. Dh' eirich Dombnuill 's na sithichean uile timchioll air, 's cha robh dannsa no ùpraid ann riamh thun an so. Ghairm an coileach, 's cha robh sgial air druis aca; 's bha Domhnull an sud leis fhein slan fallain air a' chnoc.)

Beadagan. (A' gabhail air ais an comhair a chùil mar gu 'm biodh duine 'ga ruith, 's e'g éigheach.)

'Cormag crosda crotach crom,

Buntata pronn as fhearr leis.' (Theabadh a bhualadh le bata a dh' amais Cormag air, is theich e.)

Cormag. 'S ann ort a tha 'm bial farsuinn, a luidealaich dhuibh; ach ma gheibh mise greim ort cha bhi mi fada 'ga fhuaigheal dhut, 's ni mi buntata pronn dhe d' chuid chnàmhan. (Bha feum aige-san gu 'n tug e chasan leis.) Ach co 'n duine a tha so a nuas h-ugam? Saoilidh mi gu bheil mi dianamh seors' aithneachaidh air, 's na dheaghaidh sin cha 'n eil mi cinnteach.

Domhnull. Dé 'n saoghal a th' agaibh fhein, a Chormaig?

Cormag. Saoghal riaslach mar a bh' agam riamh, 's nach leig piollain a bhail' ud thall fois leam a latha na dh' oidhche. Ach bu mhath leam fhaighinn a mach co 'n duine a tha bruidhinn rium.

Domhnull. Cha 'n 'eil sibh 'gam aithneachadh ; ach cha 'n 'eil sin 'na ioghnadh, 's nach do dh' aithnich mo bhràthair fhein mi 'n diugh 's a' mhaduinn. Nach tomhais sibh, a Chormaig, co th' ann?

Cormag. Cha 'n 'eil duine 'n taobh so Lic-na-banaraich nach aithne dhomhsa, 's bu chòr gu 'n aithneadhainn thusa ma tha thu mhuinntir na dùthcha,-rud a tha, air do Ghàidhlig,-'s na dheaghaidh sin cha dian mi mach thu. 
Domhnull. Ma ta, a Chormaig, is mise Domhnull na Croite, ged a chaill mi mo chòr air an ainm.

Cormag. Ma 's tusa Domhnull chaill thu do choir air an ainm gun teagamh, 's nach 'eil duine 's an duthaich a dh' $\mathrm{gg}$ na shean as deise 's as dìriche pearsa na thu. Ach ciod e rinn thu ris a' chnap?

Domhnull. Mata, a Chormaig, dh' fhàg mis' e far am faod sibhse fhagail ma bhios sibh fhein tapaidh.

Cormag. Dhianainn tapachd nach $\mathrm{d}^{\prime}$ rinneadh a leithid na 'n saoradh mo thapachd mi bho chnap na mollachd. Cha dian tapachd e, 's cha dian lighich e.

Domhnull. Ni daoine beag' an t-Sithein Ruaidh e, ge ta. Rinn iad dhomhs' e, 's ni iad dhuibhs' e cuideachd.

Cormag. O m' fheudail air do chridhe, Dhomhnuill, nach innis thu dhomb ciamar, 's cha ruith leam ach leam thun an t-Sithein.

Domhnull. Cha mhor a b' fheairrd thu sin. Cha bhi am fear sin fosgailte daonnan ann, 's cha 'n fhosglar e gu ceann latha 's bliadhna. 'S ann an diugh a thàinig mis' as.

Cormag. Creid thusa, ghraidhein, gu 'm bi mo shuil-sa sealltuinn gu math biorach air cnoc an aigh gu 'n tig a' chiad ghedbadh air. Ach an deaghaidh dhomh faighinn a stigh dé 's coir dhomh dhianamh? 'S ann agad fhein a tha brath air modhannan is riaghailtean an aite.

Domhnull. Cha 'n 'eil agaibh ach sibh fhein a ghiulan gu modhail iomchuidh mar gu 'm biodh sibh ann an tigh duin' uasail. Bheir iad tarruinn air dannsa. Ach tha 'n truaighe fhein air a' phort a th' aca. Cha robh tionndadh na siubhal na eile, ach an aon duan ud :-

\section{Di-luain Di-mairt.}

Di-luain Di-mairt.

Dh' iarr mis' orra 'Di-ciadaoin' a chur ris. Chòd sud riutha anabarrach, 's cha robh sian a bha 'san t-Sithean nach do thairg iad dhomb na 'n gabhainn e. Thuirt mi riutha gum b' fhearr leam a' chroit a bhi 'g am dhith na h-uile rud air an t-saoghal. Cha robh 'm facal a mach as mo bhial 'nuair a bha chroit an iomall an t-Sithein, 's mis' voL. III. 
am mullach mo shòlais a' dannsa 's a' leamadaich air an ùrlar gus an do ghairm an coileach; 's bha mis' an sud air a' chnoc annsan riochd am beil thu 'g am fhaicinn.

III

Cormag. (Air bialaobh an t-Sithein.)

Bha dùil leam riamh gu ' $n$ robh còir aig an t-Sithean a bhi fosgailte aig bial na h-oidhche, ach tha mo chnàmhan air ragadh 's mi 'nam stob 's an sgor so bho chionn thri uairean an uaireadair, 's cha 'n 'eil tuar air gu 'm fag mi e nochd. Mur a bhith an tàir a th' agam á cnap na dunach cha toireadh an saoghal orm fuireach na 's fhaide. Dé starban a tha sud? Sud e mu dheireadh an cnoc a' fosgladh. C'ait an do chuir mi 'n $t$-snathad mhor? (A' gabhail a stigh).

Murch. Cò 'm bodach grànnda gun mhodh a thainig a stigh. Cha robh guth air beannachadh no eile, ach gabhail lom direach suas dh' an aon aite as urramaiche stigh.

Sitheanach Eile. Cha b' e sin do 'n bhodach a thàinig g' ar coimhead an uiridh.

Murch. 'N e fear a phuirt?

Sitheanach. Cò eile?

Banruinn. Dh' fhaodadh nach 'eil am fear so fhein cho aineolach 's a tha choltas. Cha rachainn an urras nach 'eil làn a chlaiginn de cheòl aige. Am biodh e mi-mhodhail dhomb fhaighneachd ma tha dad a dh' edlas agaibh air ceol dannsa? (Ri Cormag.)

Cormag. 'S ann agam a tha, ma tha feum agaibh air. Siuthadadh sibhs, air dannsa 's gu 'n cluinn mi de 'n seorsa phort a tha sibh a' cleachdadh, 's gu 'n tuig mi 'n seol ciùil as fhearr a chordas ribh.

Banr. Eiribh mata thun an ùrlair agus thus', a Mhurchaidh, gabh am port is gabh gu math e.

Murch. Di-luain Di-mairt.

Di-luain Di-mairt.

Diluain Di-mairt Di-ciadaoin.

(Uile 'g a ghabhail combla 's a' dannsa.)

Cormag. Sguiribh mionaid, sguiribh, 's gu 'n eisd sibh an tionndadh ùr a bheir mise dhuibh air a' phort, 's tha mise 
meallta neo cha chuala sibhse riamh aona phort eile coltach ris. 'S cha 'n 'eil ioghnadh sam bith ged a dhianainn-sa port $a$ b' fhiach éisdeachd. Bha bhàrdachd a' ruith 's an teaghlach againn bho ghlun gu glun bho linn mo shinn-shinn-seanar. Tha 'm port sin a th' agaibh cho stubach 's nach b' fhuilear dha rud a chur ris gu snas is blas a chur air. Cuireadh sibhse 'Di-rdaoine' as a dheaghaidh, 's chi sibh gu 'm bi e dhà fheobhas.

Sitheanaich. Di-luain Di-mairt.

Di-luain Di-mairt.

Di-luain Di-mairt Di-ciadaoin Di-r-daoine.

(Ghabh iad an caothach, 's am bad a bhodaich bha iad. Chairich iad croit a' bhodaich eile air a mhuin. Ghairm an coileach, agus sud Cormag air a' chnoc 's an dà chroit air.)

\section{BOOK REVIEW}

The Place-Names of Argyll. By H. Cameron Gilures, M.D., with a short Preface from his Grace the Duke of Argyll. London: David Nutt, 1906.

One can warmly appreciate the spirit which prompted Dr. Gillies to undertake the very difficult task of explaining the place-names of Argyllshire,-a feeling that the work ought to be done and that he knew of no one else anxious or ready to do it. It is also easy to accept, in part at least, the author's judgment as to the success which crowns his efforts : ' The work is far from perfect. No person could make it perfect; and certainly no one in my position, with my poor scraps of available time, could do it better.' But it is impossible to acquiesce in the added dictum: 'I believe it is as nearly correct as any one could make it.' If this be so, one must despair of even an approach to accuracy in this branch of study. Not but that the author possesses many qualifications for such a work as this. We do not need his renewed assurance that he may 'with some justice lay claim to a very competent knowledge of old and modern Gaelic'; this has been known for many years. Dr. Gillies has the further advantage of being a native of the county, and therefore ought to be familiar with its dialect of Gaelic, including its principal localisms. He possesses a command of apt diction and phrase, a graphic and picturesque style withal. And yet the work is disappointing, and not worthy of the author's knowledge or capacity. For one thing Dr. Gillies has produced a readable book. He has stamped his own personality upon every page of it, and a delightful personality it is in many ways. He feels it necessary to apologise for his labours being presented 